Managers, $4 \mathrm{x}$ Band 5 nurses and $4 \mathrm{x}$ Assistant Practitioners. The respondents were distributed equally across the three general adult wards and the PICU. All 23 members of staff provided a score of 10 out 10 to the question about how happy they were to have a PA working on the ward. Many of the staff members provided some very positive comments on their respective views about the role of the PA at Clock View Hospital. No negative comments were provided by any members of staff.

Conclusion. It is clear from the large sample of members of staff of different grade at Clock View Hospital that were surveyed that the PA has been a warmly received and welcome addition to the inpatient team and that the PA is viewed as having become an important and valued member of the inpatient team. This provides a strong argument for both Mersey Care NHS Foundation Trust, and other mental health trusts across the U.K., to consider employing more PAs to work in their inpatient units.

Innovative psychiatry medical education initiative: empowering and supervising trainees for future teaching in psychiatry training program establishment in Somaliland

Jibril Handuleh ${ }^{1 \star}$, Victor Periera-Sanchez $\mathrm{MD}^{2}$ and Daniel Fekadu Wolde-Giorgis ${ }^{3}$

${ }^{1}$ Lecturer in Psychiatry, Amoud University School of Medicine, Psychiatry resident, Saint Paul's Hospital Millennium Medical College; ${ }^{2}$ Child and Adolescent Psychiatry fellow NYU Grossman School of Medicine, Visiting lecturer in psychiatry, Amoud University School of Medicine and ${ }^{3}$ Head of department, Visiting Professor of psychiatry, Amoud University School of Medicine, Lecturer in

Psychiatry, Institute of Psychiatry, Neurology and Psychology, King's College London

${ }^{*}$ Corresponding author.

doi: 10.1192/bjo.2021.400

Aims. Somaliland is a de facto state in the horn of Africa. It unilaterally declared independence from rest of Somalia in 1991. Medical education in Somaliland started in the year 2000.

Aim of the study is to explore the feasibility of teaching program for the country by its future potential psychiatry educators. The initiative started in 2019 to seek trainees with interest in academic psychiatry and support them with medical education skills. This is intended to prepare them for leading future teaching roles in both undergraduate and residency/fellowship in psychiatry

Amoud University wanted to empower junior doctors at the university to have teaching skills needed to set up residency program. The Somaliland government asked Ethiopian ministry of health to offer psychiatry residency program for general practitioners in Somaliland to have future residency and fellowship in psychiatry. Several psychiatry trainees worked with the visiting professor from the United Kingdom who joined Somaliland medical school as visiting professor in psychiatry

Method. The visiting professor supported the trainee in setting up a psychiatry undergraduate training curriculum in line with Somaliland medical school curriculum. Before the teaching methods were didactic and role play based. The faculty introduced different teaching methods including flipchart, small/large group teaching which was student centered education. Students received a online survey to reflect on psychiatry teaching they received. post course survey was conducted at the end of the teaching to evaluate the teaching initiative.

Result. Survey revealed interesting pattern that students preferred class room based teaching in comparison to online teaching. 90 percent of the attendees showed interest in flipchart teaching compared to didactic model. They expressed increasing understanding of the subject matter when they read and discuss among themselves instead of lectures. $70 \%$ of students prefer more clinical teaching compared to online sessions.

$52 \%$ liked the new teaching module compared to the lecturing sytle.

Conclusion. Supervision of early career psychiatrists to undertake future academic psychiatry roles is an important step in building psychiatry faculty in medical schools. As the case of Somaliland this retains trainees in teaching roles in the future to teach undergraduates mental health courses. The other benefit is empowering them to set up psychiatry training program to close the service delivery gap with skilled psychiatrists in the future. Somaliland plans to set up its psychiatry residency/fellowship programs soon after this initiative.

\section{Core psychiatry trainees views on MRCPsych course structure and delivery at East Midlands Deanery}

Asma Javed $^{1 \star}$, Hetal Acharya ${ }^{1}$ and Ian Yanson ${ }^{2}$

${ }^{1}$ Leicestershire partnership NHS Trust and ${ }^{2}$ Rampton Hospital

${ }^{\star}$ Corresponding author.

doi: 10.1192/bjo.2021.401

Aims. The RCPsych curriculum for core training in Psychiatry (2013) requires each Deanery to run regional MRCPsych teaching programme.

The East Midlands School of Psychiatry run a local MRCPsych course aimed at all core psychiatry trainees in the deanery. Before the pandemic, the course took place between two venues Nottingham and Leicester. During the pandemic, the course was delivered via Microsoft teams. We aimed to collect the feedback from trainees regarding the course to help shape the MRCPsych Course programme according to their training needs. Method. We devised an online Microsoft forms questionnaire which included:

Level of training

Number of exams passed

Relevance of MRCPsych content to clinical practice and membership exam

Usefulness of mock exams, simulation scenarios and workshops towards clinical and exam practice

Overall experience of the course

Which additional sessions they would like to be included

The effect of COVID-19 on their ability to attend in MRCPsych programme

These forms were sent to all the trainees in the region via email.

Result. Out of 44 trainees, 9 responded. $66.6 \%$ of the trainees who responded were CT1 and 33.3\% CT2. 45\% had passed Paper A and $55 \%$ had not passed any exams. $78 \%$ of them agreed and $11 \%$ strongly agreed that course was relevant to the clinical practice. $55.6 \%$ agreed that course was relevant to membership course. $44.4 \%$ agreed and $11 \%$ strongly agreed that mock exams were useful. $66.7 \%$ agreed and $11 \%$ strongly agreed that simulation case scenarios and workshops were useful for exam and clinical practice. $22.2 \%$ strongly agreed and $33.3 \%$ agreed that sessions were engaging and motivating. Overall experience of MRCPsych exam was rated as excellent (11\%), good (55\%), satisfactory (22\%) and poor (11\%).

Suggestions to add additional sessions included antiracism in psychiatry, more mock exams, practical management of cases, to organise more interactive sessions on Microsoft teams, 
in-depth coverage of exam topics, to organise full day teaching sessions instead of half day.

$33.3 \%$ of trainees commented that COVID-19 had impacted on their ability to attend the exam as initially face to face sessions were cancelled till end of May 2020 and when started there were technical issues with the online platform

Conclusion. Consider feedback received in modifying aspects of the MRCPsych course

To share the results with trainers and course tutors

Arrange relevant mock exam sessions

Include the topics suggested by trainees and improve the experience of online learning by making it more interactive

Limitations: small sample size.

\section{Reverse-mentorship of the core concepts in philosophy} and mental health: a medical education case report

Michael Jewell ${ }^{\star}$, Manzar Kamal, Richard Bayney and Heidi Hales West London NHS Trust

${ }^{\star}$ Corresponding author.

doi: 10.1192/bjo.2021.402

Aims. The aim of this medical education case report was to outline the development and outcomes of a reverse-mentorship project that enabled cross-generational collaborative learning. The project took the shape of a philosophy of psychiatry journal club facilitated by a psychiatry core trainee in west London, UK. Background. Reverse-mentorship reverses traditional roles of mentor and mentee. It is an increasingly fashionable concept in medical education. The junior mentors the senior clinician. The implicit learning outcomes include provision of a two-way learning process, development of mentoring skills for the more junior clinician and collaboration that builds social capital within the workplace. Reverse-mentorship is effective when the junior mentor is recognised for their expertise in a particular area. In this instance, the junior mentor has a special interest in the philosophy of psychiatry.

Method. Junior mentor and senior mentees formed a monthly journal club. The club tracked arguments from anti- and biological psychiatry on the meaning of mental illness. The debate offered insight into a semantic analysis of mental illness and a deeper conceptual understanding of medicine. The learning material derived from the core concepts of philosophy and mental health (Fulford et al.). The role of the mentor was to facilitate group discussion around arguments from relevant papers. A survey, adapted from a recent reverse-mentorship review article, measured the quality of educational experience for mentor and mentees.

Result. Overall, mentees (senior clinicians) agreed that the mentor (junior clinician) displayed attributes and behaviours for effective mentoring across most domains, including enthusiasm, effective communication, respect for mentee expertise and active listening to the needs of the mentee. The mentor was particularly impressed with the mentees' openness to learn new concepts and respect shown. General reflections on the experience of reverse-mentorship were positive overall. A thematic review highlighted particular aspects, including: a good way to learn a new skill and great opportunity to develop professional skills of mentoring.

Conclusion. The importance of mentoring in medical education is well established. Reverse-mentorship is a new concept that looks to harness the unique qualities of millennials, including their aptitudes for empowerment, innovation and collaboration. This medical education case report shows that an enthusiastic junior clinician can successfully pilot an educational-mentoring scheme aimed at senior clinicians. To make more explicit the intuitive benefits of reverse-mentorship, longitudinal reviews are needed. However, this case report contributes important insights into this burgeoning field of medical education.

\section{Developing resilience and promoting positive mental health strategies in university students}

Viktor Kacic ${ }^{1 \star}$, Frank Zimmerman ${ }^{1}$, Ben Milbourn ${ }^{2}$, Sonya Girdler ${ }^{3}$, Melissa Black ${ }^{2}$, Sven Bölte ${ }^{4}$ and Maya Hayden-Evans ${ }^{2}$

${ }^{1}$ Klinikum Aschaffenburg-Alzenau; ${ }^{2}$ Curtin University; ${ }^{3}$ Curtin University, Center for Neurodevelopmental Disorders at Karolinska Institutet (KIND) and ${ }^{4}$ Center for Neurodevelopmental Disorders at Karolinska Institutet (KIND), Curtin University

${ }^{*}$ Corresponding author.

doi: 10.1192/bjo.2021.403

Aims. Suicide is one of the leading causes of death in young people living in Australia, accounting for $7.3 \%$ of all deaths among individuals aged 15-19 years. Historically, high levels of suicide have been recorded in Australian university students. This project aims to develop and test a massive online course-program (MOOC) for university students, underpinned by literature and strength-based suicide prevention principles, building resilience and awareness of mental health promoting activities.

Method. A scoping review of the literature was undertaken to explore the effectiveness of current suicide prevention programs for undergraduate university students, and the effective elements contributing to the success of these programs. Six electronic databases were searched to identify relevant literature. Further, mental health consumers and university students were involved in co-producing the content of the six modules of the 'Talk-to-me' MOOC.

Result. Nine articles were included in the review, discussing four types of programs including; gatekeeping, education, promotional messaging and online consultation. It was apparent from this review that there is a significant dearth of interventions and programs currently available to reduce the risk of suicide among undergraduate students, with many of the programs having limited efficacy. Despite this, a number of program elements were identified as beneficial to preventing suicide among postsecondary students including upskilling of students, and improving resilience, and self-management. These findings and further consultation with mental health consumers and undergraduate university students underpinned the development of the content of the 'Talk-to-me' MOOC which is tailored to meet the needs of university students. The MOOC contains six modules: Mental fitness; strategies to increase mental fitness; self-harm; suicidal behaviour in young adults; interventions for suicidal behaviour; and, gatekeeper interventions. Two case study senarios depicting mental health challenges commonly experienced by yound adults portraying appropriate crisis communication skills were developed and filmed complementing the six 'Talk-to-me' modules.

Conclusion. Overall, studies included in the review provide evidence to suggest that preventative programs, incorporating an educational component may be effective to be used in the MOOC to improving help-seeking behaviours among postsecondary education students. Findings from this review have 